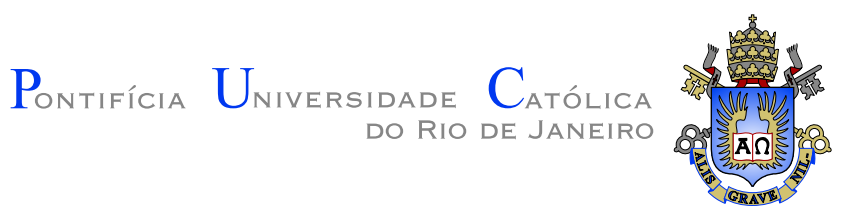

Baldoino Fonseca dos Santos Neto

\title{
JAAF: Implementando Agentes Auto-Adaptativos Orientados a Serviços
}

Dissertação apresentada como requisito parcial para obtenção do grau de Mestre pelo Programa de Pós-graduação em Informática do Departamento de Informática da PUC-Rio

Orientador: Prof. Carlos José Pereira de Lucena 


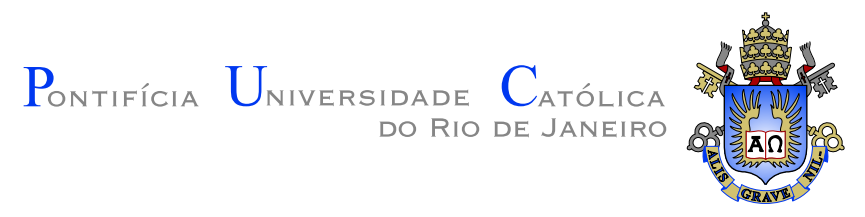

Baldoino Fonseca dos Santos Neto

\title{
JAAF: Implementando Agentes Auto-Adaptativos Orientados a Serviços
}

\begin{abstract}
Dissertação apresentada como requisito parcial para obtenção do grau de Mestre pelo Programa de Pós-graduação em Informática do Departamento de Informática do Centro Técnico Científico da PUC-Rio. Aprovada pela Comissão Examinadora abaixo assinada.
\end{abstract}

Prof. Carlos José Pereira de Lucena Orientador

Departamento de Informática - PUC-Rio

Prof. Simone Diniz Junqueira Barbosa Departamento de Informática - PUC-Rio

Prof. Viviane Torres da Silva Departamento de Ciência da Computação - UFF

Prof. José Eugenio Leal Coordenador Setorial do Centro Técnico Científico - PUC-Rio 
Todos os direitos reservados. É proibida a reprodução total ou parcial do trabalho sem autorização da universidade, do autor e do orientador.

\section{Baldoino Fonseca dos Santos Neto}

Graduou-se em Ciência da Computação na Universidade Federal de Alagoas em 2007. Atualmente atua na área de Desenvolvimento de Software Orientado a Agentes no Laboratório de Engenharia de Software (LES) da PUC-Rio.

Ficha Catalográfica

Neto, Baldoino

JAAF: Implementando Agentes Auto-Adaptativos Orientados a Serviços / Baldoino Fonseca dos Santos Neto; orientador: Carlos José Pereira de Lucena. - Rio de Janeiro : PUC-Rio, Departamento de Informática, 2010.

$106 \mathrm{f} . ; 30 \mathrm{~cm}$

1. Dissertação (mestrado) - Pontifícia Universidade Católica do Rio de Janeiro, Rio de Janeiro, 2010.

Inclui referências bibliográficas.

1. Informática - Tese. 2. Sistemas Multiagentes. 3. AutoAdaptação. 4. Mecanismos de Raciocínio. 5. Arquitetura Orientada a Serviços. 6. Web Services Semânticos.

I. Lucena, Carlos. II. Pontifícia Universidade Católica do Rio de Janeiro. Departamento de Informática. III. Título. 


\section{Agradecimentos}

Primeiramente, agradeço a Deus, por minha existência, por me conceder momentos maravilhosos.

A minha família querida, meu pai José Pedro (In memorian). A minha mãe Tercília, sempre preocupada com o meu bem-estar e esforçando-se ao máximo para me dar uma educação promissora. Ao meu irmão Walfran, pelo o seu apoio espirítual, companheiro da minha fortaleza em toda essa jornada de lutas árduas. As minhas imãs Walflania e Walfraneide.

A minha namorada Bárbara, você é a peça chave da minha vida. Esta vitória é nossa. A Dona Auxiliadora é uma satisfação, dividir esta conquista com a senhora. Obrigado pelo carinho e atenção.

Professor Carlos Lucena, meu orientador, com quem eu tive a oportunidade de aprender e interagir, tornando o trabalho mais valioso e gratificante. Professora Viviane Silva, a quem admiro, todo o seu empenho e dedicação. Professor Rodrigo Paes, foi a pessoa que primeiro me acolheu no LES e foi muito importante durante os momentos de baixo astral.

Ao pessoal de Maceió, o professor Evandro e Ig Ibert. Dedico este momento inesquecível como símbolo de amizade e consideração.

Aos colegas Elder, Manoel e Andrew, meus parceiros de trabalho desde o início do mestrado.

Aos amigos do LES, que muito contribuíram para o meu avanço profissional.

Ao departamento de informática, professores e secretaria

Por fim, a CAPES e PUC-Rio, pela ajuda financeira. 


\section{Resumo}

Neto, Baldoino; Lucena, Carlos (Orientador). JAAF: Implementando Agentes Auto-Adaptativos Orientados a Serviços. Rio de Janeiro, 2010. 106p. Dissertação de Mestrado - Departamento de Informática, Pontifícia Universidade Católica do Rio de Janeiro.

Sistemas Multiagentes Orientados a Serviços (SOMS) têm surgido visando incorporar os benefícios de duas disciplinas da Engenharia de Software: Arquitetura Orientada a Serviços e Engenharia de Software Orientada a Agentes. A primeira visa fornecer serviços fracamente acoplados que podem ser utilizados em diferentes domínios. A segunda se baseia em um novo paradigma que visa o desenvolvimento de Sistemas Multiagentes, que são compostos por entidades, chamadas agentes, com raciocínio, autonomia e pró-atividade. Um dos principais objetivos de SOMS é ajudar no desenvolvimento de sistemas orientados a serviços capazes de adaptar-se em ambientes computacionais dinâmicos, onde é necessário reagir às mudanças em seus requisitos em tempo de execução, assim como, adaptar-se eficientemente diante de erros de execução e baixa qualidade de serviço. Neste contexto, este trabalho propõe um framework ( Java self-Adaptive Agent Framework - JAAF) para implementar agentes autoadaptativos capazes de autonomamente e pró-ativamente descobrir serviços, selecionar o mais apropriado e adaptar-se caso algum problema ocorra durante a execução do serviço. A aplicabilidade do framework proposto é demonstrada através de dois estudos de caso. O primeiro é um sistema responsável por gerar mapas de susceptibilidades, ou seja, mapas que mostram locais com risco de deslizamento em determinada área. O segundo é um sistema onde o principal objetivo é satisfazer necessidades, relacionadas à viagens, de usuários.

\section{Palavras-chave}

Sistemas Multiagentes; Auto-Adaptação; Mecanismos de Raciocínio; Arquitetura Orientada a Serviços; Web Services Semânticos; 


\section{Abstract}

Neto, Baldoino; Lucena, Carlos (Advisor). JAAF: Implementing Service Oriented Self-adaptive Agents. Rio de Janeiro, 2010. 106p. MSc. Dissertation - Departamento de Informática, Pontifícia Universidade Católica do Rio de Janeiro.

Service Oriented Multi-agent Systems (SOMS) have emerged in order to incorporate the benefits of two software engineering disciplines: Serviceoriented Architecture and Agent-oriented Software Engineering. The first provides loosely coupled services that can be used within different domains. The second is based on a new software engineering paradigm that addresses the development of Multi-agent Systems, which are composed of autonomous, pro-active and reasoning entities, named software agents. One of the main goal of SOMS is to help the development of service-oriented systems able to adapt themselves on dynamic computing environments. Those systems must be able to react at runtime to changes in their requirements, as well as to efficiently accommodate for deviations from their expected functionality or quality of services. In this context, this work proposes a framework (Java selfAdaptive Agent Framework - JAAF) to implement self-adaptive agents able to autonomously and pro-actively discover services, decide about the most appropriate one and adapt themselves if they face a problem while executing the service. The applicability of the proposed framework is demonstrated through two case studies. The first is a system responsible for generating susceptibility maps, i.e., maps that show locations with landslides risks in a

given area. The second is a system where the main goal is to satisfy the users' needs related to travel.

\section{Keywords}

Multiagent System; Self-Adaptation; Reasoning Mechanisms; Service Oriented Architecture; Semantic Web Services; 


\section{Sumário}

1 Introdução $\quad 11$

1.1 Definição do Problema e Limitações das Abordagens Atuais 12

1.2 Solução Proposta e Principais Contribuições 13

$\begin{array}{lll}1.3 & \text { Organização do Documento } & 14\end{array}$

2 Fundamentação Teórica $\quad 15$

2.1 Serviços $\quad 15$

2.1.1 Web Services 16

2.1.2 Semantic Web Services 18

$\begin{array}{ll}2.1 .3 \text { OWL-S } & 19\end{array}$

2.2 JADE 26

2.3 Raciocínio Baseado em Casos 29

$\begin{array}{lll}2.4 & \text { Framework jColibri 2 } & 30\end{array}$

2.5 Algoritmo Genético 33

3 JAAF $\quad 35$

3.1 JAAF $1.0 \quad 35$

3.1.1 Detalhes do JAAF $1.0 \quad 36$

3.1.2 Hot-spots e frozen-spots do JAAF $1.0 \quad 39$

3.2 JAAF-S 40

3.3 Detalhes do JAAF-S 41

3.3.1 Collect 42

3.3.2 Analyze 44

3.3.3 Decision 54

$\begin{array}{lll}\text { 3.3.4 Effector } & 57\end{array}$

3.4 Hot-spots e Frozen-spots do JAAF-S 58

4 Cenários de Uso $\quad 60$

4.1 Escorregamentos de Massa 60

$\begin{array}{ll}4.1 .1 & \text { Idéia Principal } \\ & 60\end{array}$

4.1.2 Agentes Geradores de Susceptibilidade $\quad 61$

4.2 Agência de Viagens $\quad 74$

4.2.1 Idéia Principal $\quad 75$

$\begin{array}{ll}\text { 4.2.2 Agentes de Pacotes de Viagens } & 76\end{array}$

5 Trabalhos Relacioandos $\quad 91$

5.1 Dynamic Service-oriented Architecture (DySOA) 91

5.2 Denaro et. al. 93

5.3 Agent Service Oriented Architecture 94

5.4 Framework SEMMAS $\quad 95$

5.5 The Agent Building and Learning Environment(ABLE) 97

6 Conclusão e trabalhos futuros $\quad 98$

6.1 Principais Contribuições da Abordagem Proposta 98 
6.2 Principais Limitações da Abordagem Proposta 


\section{Lista de figuras}

2.1 Modelo da interação entre cliente, servidor e registro UDDI 17

2.2 Visão Integrada de Web Service com Semantic Web 18

2.3 Estrutura proposta de um serviço semântico em OWL-S 21

2.4 Classe Profile $\quad 22$

2.5 Referencia a arquitetura de uma plataforma de agente FIPA 26

2.6 Comportamentos do JADE 27

2.7 Ciclo RBC 29

2.8 Arquitetura do Framework jColibri 30

2.9 Arquivo databaseconfig.xm/ 31

2.10 Ciclo do Algoritmo Genético 34

3.1 Control-loop do JAAF 1.0 36

3.2 Classes do JAAF $1.0 \quad 36$

3.3 Control-loop do JAAF-S 41

3.4 Diagrama de Classes do JAAF-S 41

3.5 Atividade Collect 43

3.6 Atividade Analyze 44

3.7 OWL-S: Pacote de Viagem Nordeste Turismo 48

3.8 Fragmento de Ontologia $\quad 52$

3.9 OWL-S: Pacote de Viagem Nordeste Paraíso 53

3.10 Atividade Decision $\quad 55$

3.11 Arquivo de Configuração da Reputação 56

3.12 Atividade Effector $\quad 57$

4.1 Atividade Collect de um AGS 62

4.2 Atividade Analyze de um AGS 63

4.3 OWL-S : Modelo de Combinação Qualitativa 65

4.4 OWL-S: Modelo GeoRio 66

4.5 OWL-S: Modelo de Fator de Segurança $\quad 70$

4.6 OWL-S: Modelo de Gusmão Filho 71

4.7 Atividade Decision de um AGS $\quad 73$

4.8 Reputação $\quad 74$

4.9 Atividade Collect de um APV $\quad 77$

4.10 Atividade Analyze de um APV $\quad 77$

4.11 OWL-S do Serviço provedor do Pacote Viagem Evento 80

4.12 OWL-S do Serviço Pacote Viagem Resort 81

4.13 OWL-S do Serviço Pacote Viagem Ouro 85

4.14 OWL-S do Serviço Pacote Viagem Diamante 86

4.15 Atividade Decision de um APV $\quad 89$

4.16 Reputação $\quad 89$

5.1 Arquitetura em Alto Nível da arquitetura DySOA 92

5.2 Control-loop de auto-adaptação 93

5.3 Visão Geral do Framework $\quad 95$

5.4 Arquitetura do Framework SEMMAS 96 


\section{Lista de tabelas}

3.1 Similaridade entre o Problema Atual e o problema descrito no Caso

(1)

3.2 Similaridade a solução do Caso (1) e a Profile OWL-S da Figura 3.954

4.1 Similaridade entre o Problema Atual e o Problema do Caso (1) 67

4.2 Similaridade entre o Problema Atual e o Problema do Caso (2) 68

4.3 Similaridade a solução do Caso (1) e a OWL-S da Figura 4.5 69

4.4 Similaridade a solução do Caso (1) e a OWL-S da Figura 4.6 72

4.5 Similaridade a solução do Caso (1) e a OWL-S da Figura $4.4 \quad 72$

4.6 Similaridade entre o Problema Atual e o Problema do Caso (1) 82

4.7 Similaridade entre o Problema Atual e o Problema do Caso (2) 83

4.8 Similaridade a solução do Caso (1) e a OWL-S da Figura 4.13 87

4.9 Similaridade a solução do Caso (1) e a OWL-S da Figura 4.14 87

4.10 Similaridade a solução do Caso (1) e a OWL-S da Figura 4.12 88 